\title{
Valoración de factores de riesgo asociados a los hábitos de manejo y exposición a organofosforados y carbamatos en habitantes y trabajadores de la vereda de Bateas del municipio de Tibacuy, Cundinamarca, Colombia
}

\author{
Elkin F. Amaya V. ${ }^{1}$, Angélica M. Roa B. ${ }^{1}$, Judith E. Camacho C. ${ }^{2}$, \\ Slenavy Meneses ${ }^{3}$
}

1. Bacteriólogos y Laboratoristas Clínicos, Universidad Colegio Mayor de Cundinamarca, Bogotá, Colombia.

2. Química farmacéutica, Docente, Universidad Colegio Mayor de Cundinamarca, Bogotá, Colombia.

3. Bacterióloga. Esp. Epidemiología, Universidad Colegio Mayor de Cundinamarca, Bogotá, Colombia.

Correspondencia: slenavy@yahoo.com

Recibido 25-07-08 / Aceptado 15-08-08

\section{Resumen}

El uso de plaguicidas en la agricultura ha sido una determinante para el control de plagas. Sin embargo, estos agentes químicos empleados de forma inadecuada, especialmente por parte de los agricultores, se refleja en los efectos nocivos sobre el cuerpo humano; como intoxicación y muerte, especialmente en países subdesarrollados. El presente trabajo tuvo como objetivo valorar los factores de riesgo asociados a los hábitos de manejo y exposición a plaguicidas organofosforados y carbamatos mediante un cuestionario estructurado, la medición de la actividad colinesterásica, y parámetros hematimétricos en trabajadores y habitantes de la vereda de Bateas del Municipio de Tibacuy, Cundinamarca, Colombia, relacionados con la presencia de intoxicación.

Se encontró que los factores de riesgo relacionados más sobresalientes fueron el uso inadecuado de elementos de protección personal y la falta de evacuación previa a la fumigación. El I00\% de la población participante en el estudio tuvo niveles bajos de actividad de colinesterasa; entre 0,056 pH/hora y 0,445 pH/hora en los hombres y 0,052 $\mathrm{pH}$ /hora a 0,264 pH/hora en las mujeres, con un valor medio de 0, I $48 \mathrm{pH} /$ hora (valor de referencia: hombres de 0,855 a 0,88 I pH/hora, mujeres de 0,836 a 0,859 pH/hora). Los estudios poblacionales epidemiológicos son importantes, porque permiten valorar e identificar cualquier fenómeno que sea un riesgo para el bienestar de una población.

Palabras clave: carbamatos, colinesterasa, intoxicación, organofosforados, plaguicidas.

\begin{abstract}
Valuation of risk factors associated to the handling and exposure habits of organophosphates and carbamates in workers and inhabitants of the Bateas District, Municipality of Tibacuy, Cundinamarca, Colombia

The use of pesticides in agriculture has been a determinant for the control of plagues. Nevertheless, these chemical agents inadequate, especially by farmers, is reflected by its harmful effects on the human body, such as
\end{abstract}


poisoning and death, mostly in underdeveloped countries. The present work had as its object to value the risk factors associated with the habits of handling of and exposure to organophosphate and carbamate pesticides by means of a structured questionnaire, the measurement of the cholinesterase activity and hematohidrosis parameters in workers and inhabitants of the Bateas District, Municipality of Tibacuy, Cundinamarca, Colombia, related to the presence of poisoning.

It was found that the most outstanding risk factors were the inadequate use of elements of personal protection and the nonevacuation previous to the fumigation. $100 \%$ of the participant population in the study had low levels of cholinesterase activity; between $0.056 \mathrm{pH} /$ hour and $0.445 \mathrm{pH} /$ hour in men and $0.052 \mathrm{pH} /$ hour to $0.264 \mathrm{pH}$ / hour in women, with an average value of $0.148 \mathrm{pH} /$ hour (value of reference: men from 0.855 to $0.88 \mathrm{I} \mathrm{pH} /$ hour, women from 0,836 to $0.859 \mathrm{pH} /$ hour). Epidemiologic population studies are important, because they allow to value and to identify any phenomenon that is a risk for the well-being of a population.

Key Words: carbamates, cholinesterase, organophosphate, pesticides, poisoning.

\section{Introducción}

Los plaguicidas se han usado en la agricultura desde 1950. A pesar de que la parte clínica cuenta con algunos recursos efectivos para el manejo adecuado de los pacientes intoxicados, en muchos países en vías de desarrollo las intoxicaciones con estos agentes siguen siendo la causa de miles de muertes al año (1-3). Aunque se cuenta con medidas de seguridad en su aplicación, estos agentes han provocado efectos nocivos agudos y crónicos en el ambiente, convirtiéndose en un problema de salud pública (4).

El término plaguicida se define como el agente que combate las plagas del campo. Abarcan una gran cantidad de compuestos según su composición química y están comprendidos en 4 grupos con una acción específica (insecticidas, fungicidas, herbicidas y rodenticidas) (5). Internacionalmente está establecido que los envases y empaques de plaguicidas deben llevar una banda del color que identifique la categoría toxicológica del contenido, Tabla 1.
En el área agrícola, los organofosforados y carbamatos son los plaguicidas más usados para el control de insectos $(6,7)$. Para muchos trabajadores agrícolas, fumigadores, de industria y pobladores aledaños, la exposición a estas sustancias ocurre muchas veces desde la niñez (5-8 años) por lo que implica un factor de riesgo en el desarrollo de muchas enfermedades por intoxicación a causa de estos elementos tóxicos $(2,5)$. Los insecticidas organofosforados y carbamatos se caracterizan en la clínica por ser inhibidores de las enzimas colinesterasas, las cuales regulan el exceso de acetilcolina en el sistema nervioso $(1,3,6,7)$. Debido a su gran demanda, al igual que las intoxicaciones que ocasionan, es necesario programas de vigilancia epidemiológica, capacitación sobre el manejo de estos componentes y la adecuada atención médica para los intoxicados, entre otros.

Desafortunadamente no hay información establecida con exactitud de las intoxicaciones agudas y crónicas. Esto se debe en parte a que muchos trabajadores agrícolas viven

Tabla 1. Categorías toxicológicas según la dosis letal 50 (DL50)

\begin{tabular}{llllll}
$\begin{array}{l}\text { Categoría } \\
\text { Color banda }\end{array}$ & $\begin{array}{l}\text { Nivel de } \\
\text { toxicidad }\end{array}$ & ORAL & Sólidos & Líquidos & SÉRMICO \\
I = Roja & Extremo & $<5$ & $<20$ & $<10$ & Líquidos \\
II = Amarilla & Alto & $5-50$ & $20-200$ & $10-100$ & $<40$ \\
III = Azul & Moderado & $50-500$ & $200-2000$ & $100-1000$ & $40-400$ \\
IV = Verde & Ligero & $>500$ & $>2000$ & $>1000$ & $>4000$ \\
\hline
\end{tabular}


bajo condición de riesgo, donde muchas veces no puede saberse la incidencia real y su correlación con los niveles de exposición u otros factores que puedan relacionarse (2). Actualmente se estima que miles de personas en Colombia se dedican a la agricultura como modo de vida para satisfacer sus necesidades. Debido a esta actividad, muchos de ellos usan algún tipo de plaguicida en los cultivos donde laboran, muchas veces sin la protección adecuada, la cual puede estar influyendo en su salud y en el deterioro del medio ambiente.

\section{Materiales y métodos}

El estudio se llevó a cabo en noviembre de 2007, participaron 50 personas de forma voluntaria y con consentimiento firmado, a quienes les fue aplicado un cuestionario con el fin de recolectar información técnica sobre antecedentes laborales, condiciones de trabajo, uso anterior y actual de plaguicidas, hábitos y costumbres y listado de síntomas compatibles con intoxicación por plaguicidas inhibidores de colinesterasa. Además se les tomó una muestra de sangre para la medición de los niveles de la actividad de acetilcolinesterasa por el método electrométrico de Michel en glóbulos rojos y valoración por cuadro hemático en el sistema Advia 60. El análisis de la información se realizó a partir del programa Epi Info $6.04 \mathrm{~d}$ que permitió realizar el análisis de variables a partir del Odds Radio (OR).

Las muestras sanguíneas de acetilcolinesterasa y cuadro hemático se recolectaron en tubos con heparina y EDTA. Fueron conservadas a una temperatura de $4^{\circ} \mathrm{C}$, siendo transportados en los tiempos establecidos para el análisis de los analitos. Para la acetilcolinesterasa fue procesado antes de las primeras 48 horas según Hurtado (10), mientras que para el cuadro hemático fue realizado dentro de las 4 horas de estabilidad de la muestra para asegurar la validez de los resultados de acuerdo a la fase pre-analítica, analítica y posanalítica del control de calidad. A todos los participantes se les determinó la actividad de la acetilcolinesterasa para un total de 50 pruebas.

Aunque el valor promedio estándar de la actividad de la colinesterasa eritrocitaria en individuos normales, determinada según Michel por el método electrométrico, es de 0.570 a $0.980 \mathrm{pH} /$ hora (8), se vio apropiado, tomar valores de referencia que hayan sido establecidos por estudios previos realizados en Colombia, como los reportados por Carmona (2003) en su trabajo "Valores de referencia de la actividad de la colinesterasa eritrocitaria según técnicas de Michel y $\mathrm{EQM}^{\odot}$ en población laboral de Antioquia, Colombia"; en donde se establece como valor de referencia para la técnica de Michel en hombres de 0.855 a $0.881 \mathrm{pH} /$ hora y en mujeres de 0.836 a $0.859 \mathrm{pH} /$ hora (IC=95\%), de la población de Aburrá (Antioquia) (9), cuya altura sobre el nivel del mar es semejante a la de la vereda de Bateas del municipio de Tibacuy-Cundinamarca, Colombia (Aburrá $1540 \mathrm{~m}$, Bateas $1647 \mathrm{~m})$.

\section{Resultados}

De la población estudiada, 54\% (27) fueron hombres y el 46\% (23) mujeres. La mayoría de las personas tenían entre 40 a 51(32\%:16) ańos de edad, seguido de 52 a 63 (24\%:12) años de edad y de 64 a 75 (20\%:10) años de edad, con un promedio de 48,6 ańos. Como antecedentes encontrados se obtuvo que el promedio en años de trabajo fue de 36,2 ańos, de los cuales la media del tiempo de residencia en Bateas es de 38 años, con un mínimo de 4 años y un máximo de 85 años. La mayoría refería haber vivido en lugares previos como la Costa, Síbaté, Cúcuta, Bogotá, entre otros, por largo tiempo (6-15 años).

Entre los entrevistados el 82\% (41) manifestaron estar ante exposición por plaguicidas. El 34\% (17) de los encuestados como parte de su actividad agrícola se dedican a la aplicación de estos agentes en cultivos. También el 24\% (12) de los participantes (10 hombres y 2 mujeres), comentaron haber presentado casos previos de intoxicación

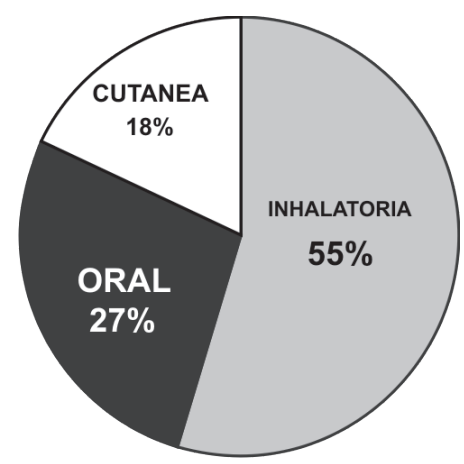

Figura 1. Distribución de las vías de intoxicación por plaguicidas (habitantes y trabajadores). Vereda de Bateas, Tibacuy, CundinmarcaColombia. 2007 
Tabla 2. Plaguicidas utilizados en fumigación en la Vereda de Bateas, Tibacuy, Cundinamarca-Colombia. 2007.

\begin{tabular}{|c|c|c|c|}
\hline $\begin{array}{l}\text { COMPUESTO } \\
\text { Organofosforado }\end{array}$ & CATEGORIA & PERSISTENCIA & $\begin{array}{l}\text { PORCENTAJE } \\
32,8\end{array}$ \\
\hline Lorsban & II & 60 a 120 días & 25,7 \\
\hline Tamaron & I & 2 días & 4,3 \\
\hline Malation & III & I semana & 1,4 \\
\hline Curacron & ॥ & 9 horas & $\mathrm{I}, 4$ \\
\hline Carbamato & & & 30,0 \\
\hline Furadan & I & 4 a 8 semanas & 28,6 \\
\hline Baygon & II & 12 horas & 1,4 \\
\hline Piretroide & & & 18,6 \\
\hline Karate & III & 12 semanas & 18,6 \\
\hline Oxalato & & & 5,7 \\
\hline Evisect & III & POCA & 5,7 \\
\hline Ditiocarbamato & & & 2,9 \\
\hline Fumigan & ॥ & I a 5 horas & 1,45 \\
\hline Manzate & III & I semana & 1,45 \\
\hline Compuestos de Cobre & & & 2,9 \\
\hline Oxicloruro de cobre & III & I hora & 1,45 \\
\hline Curzate & III & 64 horas & 1,45 \\
\hline Organoclorado & & & 2,9 \\
\hline $\mathrm{BHC}$ & II & 15 meses & 1,45 \\
\hline DDT & II & 5 días & 1,45 \\
\hline Benzimidazol & & & 1,4 \\
\hline Belante & III & 19 horas & $\mathrm{I}, 4$ \\
\hline Anilina & & & 1,4 \\
\hline Ridomil & III & 7 a 170 días & 1,4 \\
\hline Curamina & & & I,4 \\
\hline Cebo & II & POCA & I,4 \\
\hline TOTAL & & & 100,0 \\
\hline
\end{tabular}

por plaguicidas de tipo oral, inhalatorio y dérmico, mientras que el 76\% (38) no refirió haber sufrido de intoxicación, presentándose en menor proporción en mujeres, Figura 1.

En la Tabla 2 se relacionan los plaguicidas que suelen ser usados con mayor frecuencia, y en la Tabla 3 se muestran el tipo de acción de los plaguicidas utilizados en el control de plagas.

Tabla 3. Tipos de acción de los plaguicidas usados en fumigación. Vereda de Bateas, Tibacuy, Cundinamanrca-Colombia. 2007

\begin{tabular}{ll} 
CLASE & PORCENTAJE \\
Insecticida & 81,4 \\
Herbicida & 3,7 \\
Fungicida & 13,6 \\
Rodenticida & 1,2 \\
TOTAL & 100,0 \\
\hline
\end{tabular}

La información suministrada por los participantes fue posible valorar los diferentes factores de riesgo a través de la medición del OR como valor estimativo para presentar exposición o en caso más grave llegar a una intoxicación no solo por pesticidas organofosforados y carbamatos, también por otros agentes.

Como datos laborales se encontró que la mayoría de los entrevistados se dedican completamente a labores del campo, mientras que otros además tenían otro oficio en el cual se desempeñaban, Figura 2.

El análisis de riesgo mostró que la falta de uso o el uso inadecuado de los elementos de protección actúan como factor de riesgo para presentar intoxicación por plaguicidas o en el caso de exposición, Tabla 4. 
CAMPESINO

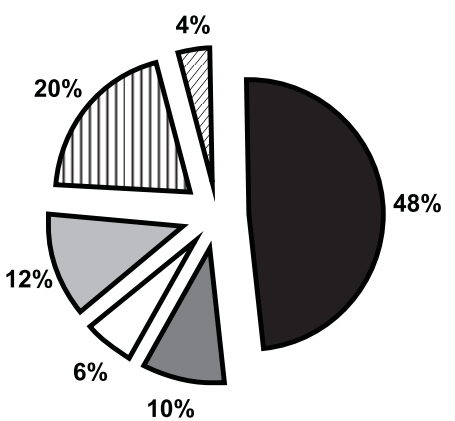

CAMPESINO Y OTRO OFICIO UII AMA DE CASA

$\square$ CAMPESINO Y ESTUDIANTE $\square$ AMA DE CASAE INDEPENDIENTE

Figura 2. Oficios laborales a los que se dedican los participantes. Vereda de Bateas, Tibacuy, Cundinamarca-Colombia 2007.

\section{FRECUENCIA DE APLICACIÓN DE PLAGUICIDAS EN CULTIVOS}

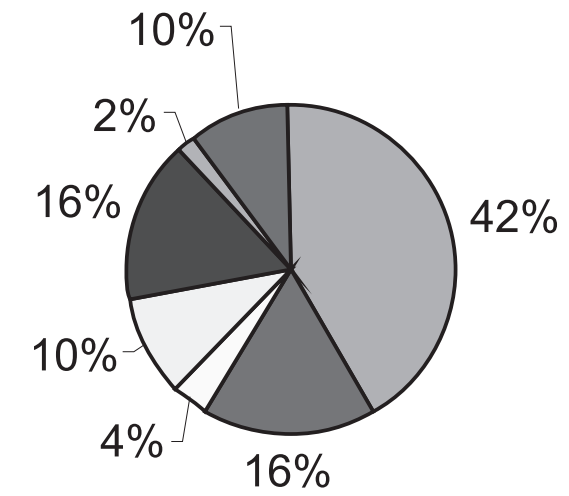

\begin{tabular}{ll} 
I vez por semana & I vez al mes \\
\hline veces por semana & 2 veces al mes \\
\hline $\begin{array}{l}\text { Más de } 2 \text { veces por } \\
\text { semana }\end{array}$ & Semestral \\
\hline
\end{tabular}

Figura 4. Periodos de fumigación con plaguicidas en los cultivos. Vereda de Bateas, Tibacuy, Cundimanrca-Colombia, 2007.

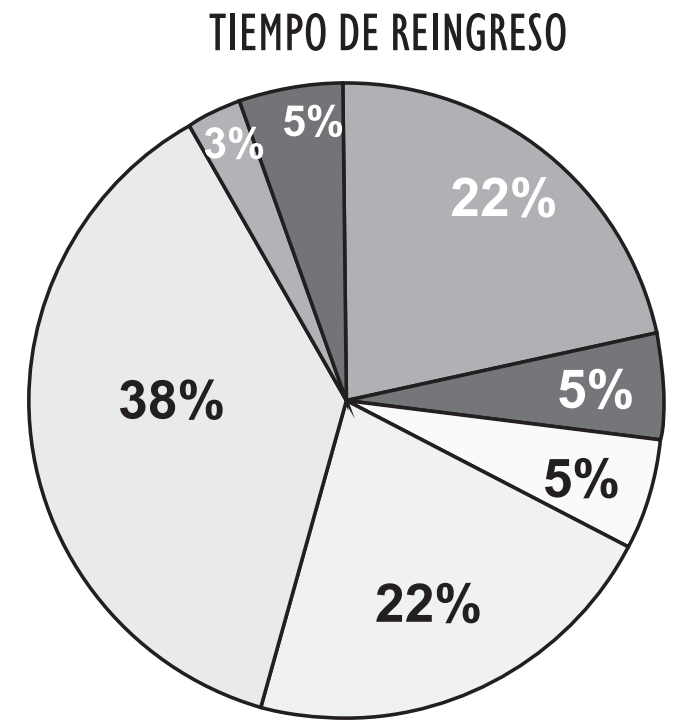

Menor de 30 minutos Mayor de I hora

A los 30 minutos

Al día siguente

Mayor de 30 minutos

A los tres días

A los ocho días

Figura 3. Tiempo de reingreso posterior a la fumigación con plaguicidas. Vereda de Bateas Tibacuy, Cundinamarca-Colombia, 2007.

El promedio en horas laborales fue de 9-10 horas con una frecuencia de trabajo de varios días (valor bimodal 7 y 6 días) de los cuales la mayoría de ellos no utilizan de forma adecuada o no utilizan los elementos de protección, Tabla 5.

Durante la aplicación se encontró que 36 de los encuestados sí evacuan la zona del cultivo antes de fumigar, 11 refirieron no salir en el momento de la fumigación, mientras que 3 no respondieron. Siendo este un factor de riesgo para estar expuesto a plaguicidas y a su vez para intoxicarse. Del tiempo de reingreso posterior a la fumigación, se encontró que la mayoría de veces

Tabla 4. Factores de riesgo y factores protectores relacionados con la exposición e intoxicación por pesticidas. Vereda de Bateas, Tibacuy, Cundinamarca-Colombia 2007.

\begin{tabular}{lll} 
FACTORES DE RIESGO & OR EXPOSICIÓN & OR INTOXICACIÓN \\
Uso inadecuado de elementos de protección personal. & 5.71 & 1.05 \\
No evacuación previa a la fumigación. & 1,36 & 5,72 \\
Tiempo de reingreso posterior a la fumigación. & 1,45 & 1,40 \\
Cambio de ropa después de la jornada laboral. & 1,42 & 1,10 \\
Fumar & ---- & 2,33 \\
\hline FACTORES PROTECTORES & OR EXPOSICIÓN & OR INTOXICACIÓN \\
El baño personal al finalizar la actividad laboral. & 0,69 & 0,45 \\
Fumigación realizada una vez por semana. & 0,29 & I,53 \\
\hline
\end{tabular}


Tabla 5. Utilización de elementos de protección. Vereda de Bateas, Tibacuy, Cundinamarca-Colombia 2007.

\begin{tabular}{lll} 
ELEMENTOS DE PROTECCIÓN & PERSONAS & PORCENTAJE \\
ADECUADO & 4 & 8 \\
INADECUADO & 29 & 58 \\
NO USA & 16 & 32 \\
NO RESPONDE & 1 & 2 \\
TOTAL & 50 & 100 \\
\hline
\end{tabular}

suele realizarse al día siguiente con un factor de riesgo de exposición y de intoxicación, Figura 3.

Sobre la frecuencia de aplicación, generalmente suele realizarse una vez por semana por medio de aspersores, aunque algunos lo aplican de forma manual con instrumentos caseros, Figura 4. Siendo este un factor protector en la exposición por pesticidas pero un factor de riesgo para intoxicarse, Tabla 4.

Sobre el modo de almacenamiento se encontró que los participantes guardan los insecticidas en cuarto aislado (50.9\%), de los cuales el $18.2 \%$ los dejan de forma libre (frascos), el 20\% lo depositan en canecas, el 10,9\% en bolsas plásticas y el $1,8 \%$ en costales. Otras formas de almacenaje encontrados fue en campo abierto (36.4\%) donde el $14,5 \%$ esta en canecas, el $10,9 \%$ en bolsas, el $5.5 \%$ en costales, el $5.5 \%$ de forma libre y dentro de la casa $(12,7 \%)$ siendo el $5.5 \%$ de forma libre, 3,6\% en costales, bolsas plásticas $1,8 \%$ y canecas $1,8 \%$.

Entre los hábitos de trabajo sólo el 8\% (4) de los entrevistados fuma, haciéndolo en donde residen (1), donde residen y laboran (1), en un lugar apartado (1) o en los tres lugares (1) demostrando ser esta variable un potencial factor de riesgo para presentar una intoxicación por pesticidas, Tabla 4. En el consumo de alimentos se encontró que los encuestados lo hacen en gran parte donde residen 68\% (34), donde laboran $16 \%$ (8) y en lugares apartados $16 \%(8)$, pero hay que tener en cuenta que el lugar donde residen también es su propia área de trabajo. Del mismo modo en la parte del baño personal y cambio de ropa después de la jornada laboral se encontró que suele realizarse generalmente 1 o 2 horas posteriores a su actividad, Tabla 5. Siendo este tipo de exposición un factor protector de exposición e intoxicación para el baño personal y un factor de riesgo de exposición e intoxicación para el cambio de ropa, Tabla 4.

Los síntomas reportados con mayor frecuencia fueron: adormecimiento y hormigueo en extremidades (9.9\%), dolor de cabeza $(9,6 \%)$, visión borrosa $(9,6 \%)$, dolor muscular $(8,8 \%)$, debilidad $(8,5 \%)$, espasmos musculares (7\%), ojos irritados $(6,6 \%)$, mareo o vértigo $(4,4 \%)$, irritación de garganta $(4,0 \%)$, sudoración anormal $(4,0 \%)$, lagrimeo $(3,7 \%)$, náuseas $(3,7 \%)$, dolor abdominal $(3,3 \%)$, flemas $(3,3 \%)$, confusión mental $(3,3 \%)$, temblores $(2,2 \%)$, diarrea $(2,2 \%)$, marcha alterada y perdida del conocimiento $(1,8 \%)$, salivación $(1,8 \%)$, dificultad respiratoria (1,5\%), vómito $(0,7 \%)$.

Tabla 6. Rangos de actividad de la acetilcolinesterasa (habitantes y trabajadores). Vereda de Bateas, Tibacuy, Cundinamarca-Colombia 2007.

$\begin{array}{lllllll}\begin{array}{l}\text { ACTIVIDAD } \\ \text { ACETILCOLINESTARASA } \\ \text { (Delta pH/hora) }\end{array} & \begin{array}{l}\text { NÚMERO } \\ \text { PERSONAS }\end{array} & \% & \text { HOMBRES } & \% & \text { MUJERES } & \% \\ 0,049-0,106 & 16 & 32 & 7 & 25,9 & 9 & 39,1 \\ 0,107-0,163 & 12 & 24 & 6 & 22,2 & 6 & 26,1 \\ 0,164-0,220 & 15 & 30 & 8 & 29,6 & 7 & 30,4 \\ 0,221-0,277 & 4 & 8 & 3 & 11, I & 1 & 4,3 \\ 0,278-0,334 & 1 & 2 & 1 & 3,7 & 0 & 0,0 \\ 0,335-0,391 & 1 & 2 & 1 & 3,7 & 0 & 0,0 \\ 0,392-0,448 & 1 & 2 & 1 & 3,7 & 0 & 0,0 \\ \text { TOTAL } & \mathbf{5 0} & 100 & \mathbf{2 7} & 100 & \mathbf{2 3} & \mathbf{1 0 0}\end{array}$

Valores de referencia: hombres de 0.855 a $0.88 \mathrm{\Lambda} \mathrm{AH} /$ hora; mujeres de 0.836 a $0.859 \mathrm{~A} \mathrm{pH} /$ hora $(\mathrm{IO})$. 
Sobre la manifestación de estos síntomas, el 96\% (48) de los participantes presentaba más de un síntoma simultáneamente desde dos e inclusive más de nueve, mientras el 4\% (2) refería presentar sólo uno. Del mismo modo el 42\% (21) manifestó presentarlos antes y después de su actividad laboral, el 32\% (16) anotó presentarlos después de su jornada laboral, el 14\%(7) refirió presentarlos antes y el $12 \%$ (6) no respondió.

Entre los resultados obtenidos de colinesterasa eritrocitaria se encontraron variaciones de baja actividad de acuerdo al valor en $(\Delta)$ de $\mathrm{pH} /$ hora para hombres y mujeres con valores mínimos y máximos de $0,056 \mathrm{pH} /$ hora y 0,445 $\mathrm{pH} /$ hora (hombres) con un valor medio de $0,169 \mathrm{pH} /$ hora, desviación estándar $0,098 \mathrm{pH} /$ hora y coeficiente de variación 0,578 ; y 0,052 pH/hora y 0,264 pH/hora (mujeres) con valor medio $0,124 \mathrm{pH} /$ hora, desviación estándar 0,056 pH/hora y coeficiente de variación 0,455. El valor medio de la actividad colinesterásica fue de 0.148 $\mathrm{pH} /$ hora. A pesar de que mayoría de estos pacientes no eran fumigadores presentaron síntomas de acuerdo a lo hallado en los cuestionarios, Tabla 6.

\section{Discusión}

A pesar de que actualmente existen reglamentaciones sobre el uso de plaguicidas, vale la pena mencionar que algunos como el Manzate, el BHC, el DDT y el Benlate, cuyas licencias de venta han sido canceladas según la resolución 5052 de 1989 del ICA, resolución 10255 de 1993 del Ministerio de Salud, resolución 001973 de 2004 del ICA y resolución 4863 de 1989 del ICA, respectivamente, aún se siguen utilizando.

A través de los factores de riesgo se pudo encontrar que los participantes se encuentran a diario en presencia de riesgos químicos por exposición a plaguicidas que interaccionan con el medio ambiente y su persistencia puede influir en una población sin necesidad de manipular estos agentes directamente. También se debe tener en cuenta el ambiente de trabajo, las condiciones de organización laboral, los malos hábitos y demás aspectos personales del trabajador y su entorno social, que intervienen en la problemática mencionada.

Con respecto a los valores hallados por el OR para los factores de riesgo se estima que los diferentes casos como el uso inadecuado de elementos de protección personal existe el riesgo de exposición mayor que puede conllevar a una intoxicación. La no evacuación previa a la fumigación indica un fenómeno de riesgo para sufrir una intoxicación más inmediata debido a las dosis a las que pueden estar expuestos y a la susceptibilidad de cada uno de los trabajadores; en el cambio de ropa, la presencia de la exposición e intoxicación están presentes por el uso de ropas contaminadas con el plaguicida, ya que al permanecer por mucho tiempo con este elemento puede crear un foco de contaminación para el trabajador y para quienes lo rodean. El fumar puede ser un catalizador para la inhalación de vapores o aerosoles que contengan plaguicidas y favorezcan la presencia de intoxicación. Estas variables, en comparación con el trabajo de Martha Palacios y Oscar Cárdenas $(6,11)$ donde se evaluó la presencia de exposición a plaguicidas por hábitos de trabajo, fueron similares a lo hallado en este estudio.

Los valores de OR son más bajos cuando se tienen en cuenta los factores de protección, como el baño personal posterior a la finalización de la jornada laboral, lo que reduce la probabilidad de intoxicación en las personas. La fumigación realizada una vez por semana ayuda a reducir en parte la exposición por plaguicidas, pero no indica que pueda sufrir una intoxicación a largo plazo.

Desafortunadamente no existen registros de control toxicológico de plaguicidas sobre todo a nivel crónico y no son claras las intervenciones del Plan de Atención Básico con respecto a la atención primaria para el manejo de casos de intoxicación por dichas sustancias en zonas rurales. Lo anterior quizás debido a múltiples factores sociales y económicos, situación que es crítica, ya que la falta de capacidad económica para sufragar el precio de tratamientos y controles médicos poniendo en riesgo su salud es otro aspecto importante.

Sobre los valores obtenidos de la prueba de acetilcolinesterasa por el método de Michel con respecto a los de referencia mencionados anteriormente se halló que se encontraban disminuidos en los participantes voluntarios. Cabe anotar que los valores establecidos en el estudio de Carmona se realizaron a partir de personas laboralmente activas que no empleaban plaguicidas inhibidores de colinesterasa o con labores independientes a las del campo en Colombia (9), siendo aceptable estos rangos de referencia para ser aplicados en la población 
Tabla 7. Parámetros hematimétricos encontrado en los participantes (habitantes y trabajadores). Vereda de Bateas, Tibacuy, CundinamarcaColombia 2007.

\begin{tabular}{ll} 
PARÁMETROS & FRECUENCIA \\
HEMATIMÉTRICOS & $\%$ \\
\hline Leucopenia & 2 \\
Neutrofília & 2 \\
Eosinofília & 12 \\
Trombocitosis & 4 \\
\hline
\end{tabular}

de Bateas, quienes han estado expuesto a estos tipos de agentes por muchos ańos.

Teniendo en cuenta los valores obtenidos de la acetilcolinesterasa, es posible que los pacientes incluyendo los que tuvieron valores muy bajos de actividad de la misma, sin presentar complicaciones graves y con variabilidad de los mismos de acuerdo a las edades, puede deberse a la presencia de tolerancia biológica desarrollado por los mismos. Por lo que su determinación es importante como índice de exposición en personas que están en contacto con plaguicidas organofosforados y carbamatos por largo tiempo.

Esta situación se vería reflejada a partir de la variabilidad biológica, la cual se observaría de modo intra-individual e inter-individual (12), donde las variables de exposición continua a plaguicidas puede variar durante la vida del individuo, por lo que sería necesario una participación más grande de la población para determinar la significancia de este fenómeno a través de modelos simples y sencillos, pero representativos para aportar datos más eficaces.

Se puede considerar que la situación de exposición crónica en los participantes puede deberse a las prácticas de actividad agrícola relacionadas con el incumplimiento en normas de seguridad en el manejo de plaguicidas, los malos hábitos en el consumo de alimentos sin la adecuada higiene, entre otros; que se evidenciaron con los bajos niveles de actividad colinesterásica encontrados. La persistencia de los plaguicidas en los cultivos y en el ambiente puede variar por la condición de los suelos: anaerobiosis terrestre, el $\mathrm{pH}$ y la bioacumulación de estos agentes tóxicos por absorción de las plantas, que finalmente llega al contacto humano (13).

Del mismo modo la presencia de síntomas reportados en este estudio de acuerdo con exposición crónica a plaguicidas no puede definirse en su totalidad debido a la inespecificidad de los mismos, ya que no existe una clasificación a nivel crónico de este evento en comparación con una exposición o intoxicación aguda el cual muestra una sintomatología característica de la anomalía. Quedaría en incertidumbre si la posibilidad de este fenómeno pueda deberse a una adaptación individual ante la exposición por plaguicidas o al inicio de un desarrollo de resistencia genética que esté siendo trasmitida a través de las generaciones de campesinos que por largo tiempo han llevado este estilo de vida; esto asociado a un lento proceso evolutivo como se ha presentado en diferentes plagas que han desarrollado esta variación de resistencia (14).

Aunque los resultados del cuadro hemático mostraron pocas anormalidades, no evidenciaron ser significativas para asociarlo como una consecuente de exposición o intoxicación por plaguicidas inhibidores de colinesterasa a pesar de lo expresado por otros autores (15-17) sobre la asociación de los parámetros hematimétricos como leucopenias, eosinófilia, linfocitosis y trombocitopenia en procesos agudos de intoxicación, muchas veces sin justificar la afirmación sobre esta variable con la problemática antes mencionada.

Lo anterior puede deberse a que cuando la inhibición es lenta, como ocurre en casos de exposición crónica a bajas concentraciones, la correlación con la enfermedad puede ser baja o nula (18), por lo que sería recomendable realizar estudios de medidas de control médico y promoción en salud sobre el impacto de plaguicidas a nivel crónico, ambiental, ocupacional y de seguridad sanitaria para la población de Bateas. Cabe mencionar que la falta de participación poblacional puede ser un factor por el cual las campañas de salud no logran ser efectivas, concurriendo así en una falla de los aportes en salud, ya que a veces la no intervención es debido a la negativa de la propia gente.

De otro lado, los avances científicos con respecto a la exposición por plaguicidas son muy diversos, pero debemos tener en cuenta que a pesar de la legislación vigente, muchas personas del campo viven en riesgo de una exposición por plaguicidas y la forma en que afecta a 
cada individuo es diferente, variando en la presentación de síntomas desde un modo leve hasta presentar una complicación.

La aplicación del cuestionario estructurado permitió identificar los factores de riesgo y de protección para la población referida. De acuerdo a las recomendaciones e intervenciones realizadas en la población de Bateas, y según los hallazgos encontrados, es importante resaltar la necesidad de la vigilancia en salud a nivel toxicológico poblacional sobre plaguicidas, dado su impacto a nivel de salud pública, ya que permiten valorar e identificar cualquier riesgo para una población, mejorar las condiciones laborales y de vida de los habitantes de las zonas rurales. $\mathrm{NO}$

\section{Referencias}

1. Eddleston M, Eyer P, Worek F, Mohammed F, Senarathna L, Meyer L, et al. Differences between Organophosphorus Insecticides in Human Self-Poisoning. Lancet. 2005;366:1452-1459.

2. Antonijevic B, Stojiljkovic M. Unequal Efficacy of Pyridinium Oximes in Acute Organophosphate Poisoning. Clin Med Res. 2007;5:71-82.

3. Roberts D, Karunarathna A, Buckley N, Manuweera G, Rezvi MH, Eddleston M. Influence of Pesticide Regulation on Acute Poisoning Deaths in Sri Lanka. Bull World Health Organ. 2003;81:789-798.

4. Carmona J. Correlación y conversión entre valores de colinesterasa eritrocitaria medida con las técnicas de Michel y EQM ${ }^{\circledast}$. Biomédica. 2006;26:16-30.

5. Jameson R, Seidler F, Slotkin T. Nonenzymatic functions of acetylcholinesterase splice variants in the developmental neurotoxicity of organophosphates: chlorpyrifos, chlorpyrifos oxon, and diazinon. Environ Health Perspect. 2007;115:65-70.

6. Palacios M. Aplicación de un instrumento para evaluar exposición a plaguicidas organofosforados, efectos agudos y subagudos en la salud de trabajadores agrícolas. Rev Fac Med UNAM. 2003,46:22-27.
7. O’Malley MA, McCurdy SA. Subacute poisoning with phosalone, an organophosphate insecticide. West J Med. 1990;153:619-624.

8. Peña A, Pérez T. Mahecha M. Intoxicación con plaguicidas organofosforados, diagnóstico y tratamiento. ANDI. 1996; 24-26.

9. Crarmona J. Valores de referencia de la actividad de la colinesterasa eritrocitaria según las técnicas de Michel y EQM ${ }^{\circledast}$ en población laboral de Antioquia, Colombia. Rev Panam Salud Publica/Pan Am J Public Health. 2003;14:316-324

10. Hurtado J, Lopez M, Monteoliva M. Estabilidad de la actividad colinesterasica en sangre humana. Departamento de bioquímica. Instituto Lopez Neyra de Parasitologia. C.S.I.C. 1989; 31-36.

11. Cárdenas O, Silva E, Morales L, Ortiz J. Estudio epidemiológico de exposición a plaguicidas organofosforados y carbamatos en 7 departamentos colombianos 1998-2001. Biomédica. 2005;25:170-180

12. Repetto M, Martínez D, Sanz P. Actualización de la toxicología de los plaguicidas. Toxicología avanzada. Editorial Díaz Santos. Madrid; 1995. pg.557-600.

13. Servicio Nacional de Aprendizaje-SENA, Cámara Procultivos de la Asociación Nacional de Empresarios de Colombia-ANDI. Colombia; 2007. pg. 68-69.

14. Murillo A. Resistencia a los insecticidas. Manejo responsable de productos para la protección de cultivos. Colombia: Servicio Nacional de Aprendizaje-SENA, Cámara Procultivos de la Asociación Nacional de Empresarios de ColombiaANDI; 2007.pg. 186-189

15. Córdoba D. Toxicología. Manual Moderno. 2006. pg. 124-125 y 139-145.

16. Sarabia C, Negrón L, Meléndez M. Estudio bioquímicoclínico en personas ocupacionalmente expuestas a la acción de agroquímicos y efectos de su uso frecuente sobre la salud. Ciencia e investigación. 1998;1:6-15.

17. Diaz V, Pistilli N, Guillén R, Melgarejo M, Velásquez G. Valores hematologicos en individuos expuestos accidentalmente a insecticidas organofosforados. Departamento de bioquímica. Universidad Nacional de Asunción. 2002.

18. Robert L. Enciclopedia de salud y seguridad en el trabajo. Ministerio de Trabajo y Asuntos Sociales. España. 2001; 26-28.

www.unicolmayor.edu.co 